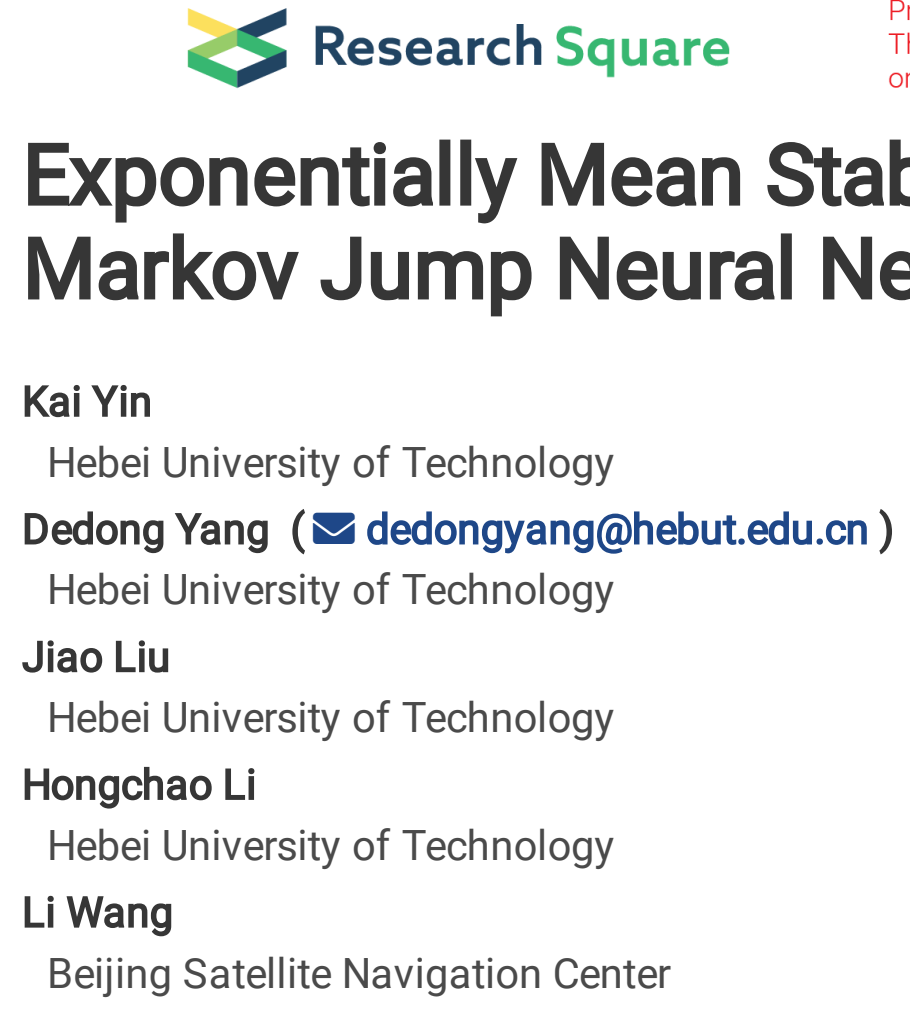

\author{
Kai Yin \\ Hebei University of Technology \\ Dedong Yang ( $\square$ dedongyang@hebut.edu.cn ) \\ Hebei University of Technology \\ Jiao Liu \\ Hebei University of Technology \\ Hongchao Li \\ Hebei University of Technology \\ Li Wang \\ Beijing Satellite Navigation Center
}

\title{
Exponentially Mean Stability Analysis of Positive Markov Jump Neural Networks with Time Delay
}

\section{Research Article}

Keywords: Positive Markov jump neural networks, Linear programming, Inequality technique, Exponentially mean stability

Posted Date: October 22nd, 2021

DOI: https://doi.org/10.21203/rs.3.rs-942126/v1

License: (c) (i) This work is licensed under a Creative Commons Attribution 4.0 International License.

Read Full License 


\title{
Exponentially mean stability analysis of positive Markov jump neural networks with time delay
}

\author{
Kai Yin ${ }^{1,2}$. Dedong Yang ${ }^{1,2}$. Jiao Liu ${ }^{1,2}$. \\ Hongchao $\mathbf{L i}^{1,2}$. Li Wang ${ }^{3}$
}

the date of receipt and acceptance should be inserted later

\begin{abstract}
A new result for the analysis of positive Markov jump neural networks (PMJNNs) with time delay is described in this paper. By rewriting the PMJNNs with time delay in both continues-time and discrete-time domains into equivalent positive neural networks(PNNs) and analyzing their stability issues, two delay-dependent sufficient conditions are presented to ensure that the continuous-time and the discretetime PMJNNs with time delay are exponentially mean stable(EMS) through using the inequality technique. All conditions obtained in the paper are in terms of standard linear programming, which reduces the conservatism. Finally, two numerical examples are provided to verify the validity of our results.
\end{abstract}

Keywords Positive Markov jump neural networks · Linear programming · Inequality technique $\cdot$ Exponentially mean stability

\section{Introduction}

Research on neural networks(NNs) has attracted substantial attention of scholars over that last few decades by the fact that they can be applied far and wide in many prac-

Kai Yin

201912801004@stu.hebut.edu.cn

Dedong Yang $(\bowtie)$

dedongyang@hebut.edu.cn

Jiao Liu

jiaoliu@hebut.edu.cn

Hongchao Li

hcli@hebut.edu.cn

Li Wang

1176018975@qq.com

${ }^{1}$ School of Artificial Intelligence, Hebei University of Technology, Tianjin, China

${ }^{2}$ Control Engineering Technology Research Center, Hebei University of Technology

${ }^{3}$ Beijing Satellite Navigation Center, Beijing, China 
tical fields, such as pattern recognition [1], signal processing [2], finance [3] and so forth. It is public knowledge that many practical systems only experience nonnegative variables(see, for example, [4-6]), which gives rise to exploration of positive neural networks(PNNs). By using some novel comparison techniques, the work in [7] has analysed the global exponential stability of PNNs with time-varying delay and a testable condition has been derived to guarantee the uniqueness of positive equilibrium point. The authors in [8] have finished off the work where the exponential stability issue of PNNs in bidirectional associative memory(BAM) model with multiple time-varying delays was solved. The filter design with $l_{1}$-gain disturbance attenuation performance has been implemented for discrete-time PNNs in [9].

On another research front, Markov jump systems(MJSs) [10-13] that can be used to explore the practical system with random changes in structure and parameters were a special class of stochastic hybrid systems. In fact, there exist random mutations in structure for NNs due to component failures, sudden environmental disturbances or changing subsystem interconnections, which can be modeled as a Markov model and brings about the discussion of Markov jump neural networks(MJNNs). Up to date, some substantial results of MJNNs have been explored in a large body of literature on stability analysis $[14,15]$, synchronization control [16-18], state estimation [1921], filter design [22-24] and so forth. Very recently, some initial efforts have been committed to the research of positive Markov jump neural networks(PMJNNs) [25]. The work in [25] has investigated the finite-time stabilization of uncertain PMJNNs and a finite-time stabilizable controller has also been designed, where all obtained conditions exist in form of linear matrix inequality(LMI). As far as we know, only a few limited works were devoted to PMJNNs and the existing results are highly conservatism. How to reduce the conservatism of the results may make the stability analysis of the PMJNNs with time delay more complex and the corresponding work remains to be studied, which is more challenging and meaningful.

In accordance with the analysis of the aforementioned results, the main purpose of this paper is to study the exponentially mean stability of the PMJNNs with time delay and to obtain less conservative conditions. The major contributions of this paper are as below:

(1)For the first time, the linear programming(LP) method is applied to explore the stability issues of PMJNNs with time delay in both continues-time and discrete-time domains. Compared with the LMI method in [25], it is on reducing computational complexity that the LP method is obviously better than the LMI method and the relation between the LP method and the LMI method has been studied in detail in [26].

(2)By virtue of the augmentation system approach, the PMJNNs with time delay in this paper are rewritten as the augmented systems and the equivalence between the PMJNNs with time delay and the augmented systems are further discussed. And by means of the inequality technique, two delay-dependent sufficient conditions that make PMJNNs with time delay exponentially mean stable(EMS) are provided. Besides, numerical examples further verify that the stability of the PMJNNs is related to the size of time-delay.

The rest of the paper is as follows. In Section II, some essential Lemmas, Definitions, Assumption and the system formulae are given. The main results are discussed 
in Section III and verified by numerical examples in Section IV. Finally, the conclusion of the paper is given in Section V.

Notations: The set of real numbers and n-dimensional (positive) Euclidean space are expressed as $R, R^{n}\left(R_{+}^{n}\right)$, respectively. $R^{n \times m}$ denotes the space of $n \times m$ matrix. $A \succeq 0$ and $A \preceq 0$ indicate that all elements of matrix $\mathrm{A}$ are nonnegative and nonpositive. $A \succ 0(A \prec 0)$ means that every element of matrix $A$ is positive (negative). $a_{i j}$ represents the element of the $i$-th row and $j$-th column of the matrix $A=\left\{a_{i j}\right\}$. $E\{$.$\} stands for the mathematical expectation and \otimes$ denotes Kronecker product. The 1-norm and the transposition of the matrix $A$ are described as $\|A\|_{1}$ and $A^{T}$, respectively. $I_{n}$ denote the identity matrix of $n \times n$.

\section{Problem statments and preliminaries}

For a given probability space $(\Omega, \mathscr{F}, \mathscr{P})$, we consider a class of continuous-time PMJNNs with time delay as follows

$$
\Sigma_{1}:\left\{\begin{array}{l}
\dot{x}(t)=-D_{r(t)} x(t)+A_{r(t)} f(x(t))+B_{r(t)} g(x(t-\tau))+J_{r(t)} \\
x(\theta)=\phi(\theta), \theta \in[-\tau, 0]
\end{array}\right.
$$

where $x(t) \in R_{+}^{n}$ is the system state associated with n-neurons. $\{r(t), t \geq 0\}$ represent a Markov process, takeing values in the set $\mathbb{M}=\{1,2, \ldots, M\} . \Upsilon=\left\{\lambda_{i j}\right\}$ is transition rate matrix of Markov process $\{r(t), t \geq 0\}$ and the rates is characterized as

$$
\mathscr{P}\left\{r_{(k+\Delta)}=j \mid r_{t}=i\right\}= \begin{cases}\lambda_{i j} \Delta+o(\Delta), & i \neq j \\ 1+\lambda_{i j} \Delta+o(\Delta), i=j\end{cases}
$$

where $\Delta>0, \lim _{\Delta \rightarrow 0}(o(\Delta) / \Delta)=0, \lambda_{i j} \geq 0(i, j \in \mathbb{M}, i \neq j)$, and $\lambda_{i i}=-\sum_{j=1, j \neq i}^{M} \lambda_{i j}$.

For $r(t)=i \in \mathbb{M}$, the $i$ th mode of the system $\Sigma_{1}$ are described by $D_{i}, A_{i}, B_{i}, J_{i}$. $D_{i}=\operatorname{diag}\left\{d_{z}^{i}\right\} \in R^{n \times n}, A_{i} \in R^{n \times n}$ and $B_{i} \in R^{n \times n}$ are constant matrices, where $d_{z}^{i}>0$ is the self-feedback term, $z \in[1,2, \ldots, n] . f(y())=.\left[f_{1}\left(x_{1}(.)\right), f_{2}\left(x_{2}(.)\right), \ldots, f_{n}\left(x_{n}(.)\right)\right]^{T}$ and $g(x())=.\left[g_{1}\left(x_{1}(.)\right), g_{2}\left(x_{2}(.)\right), \ldots, g_{n}\left(x_{n}(.)\right)\right]^{T}$ are two neuron activation functions. $J_{i}$ is the external positive input vector. $\phi(\theta)$ is the initial value with $\phi(\theta)=\phi(0)$ for $\theta \in[-\tau, 0]$ and $\tau$ is a given constant time delay.

Concerning the discrete-case, we examine a kind of discrete-time PMJNNs with time delay as below

$$
\Sigma_{2}:\left\{\begin{array}{l}
x(k+1)=D_{r(k)} x(k)+A_{r(k)} f(x(k))+B_{r(k)} g(x(k-\beta))+J_{r(k)} \\
x(\theta)=\varphi(\theta), \theta \in[-\beta, 0]
\end{array}\right.
$$

where the system state $x(k) \in R_{+}^{n}$ is controlled by n-neurons. Markov sequence $\{r(k), k \geq$ $0\}$ take values in set $\mathbb{N}=\{1,2, \ldots, N\}$ with transition probability matrix $\Pi=\left\{\pi_{p q}\right\}$ defined by

$$
\mathscr{P}_{r}\{r(k+1)=q \mid r(k)=p\}=\pi_{p q}
$$

Clearly, for all $p, q \in \mathbb{N}, \pi_{p q} \in[0,1]$, and for all $p \in \mathbb{N}, \sum_{q=1}^{M} \pi_{p q}=1$.

For $r(k)=p \in \mathbb{N}, D_{p}, A_{p}, B_{p}$ and $J_{p}$ indicate the corresponding matrixes of the $p$ th mode of the system $\Sigma_{2} . \varphi(\theta)$ is the initial value with $\varphi(\theta)=\phi(0)$ for $\theta \in[-\beta, 0]$ 
and $\beta$ is a given constant time delay.

Assumption 1. we assume that the activation function $f_{u}($.$) and g_{u}($.$) are continuous$ and bounded on $R_{+}$for $u \in[1,2, \ldots, n]$ such that the following conditions

$$
0 \leqslant \frac{f_{u}(x)-f_{u}(y)}{x-y} \leqslant L_{u f}, 0 \leqslant \frac{g_{u}(x)-g_{u}(y)}{x-y} \leqslant L_{u g}
$$

holds for $x, y \in R^{+}, x \neq y$. We describe

$$
L_{f}=\operatorname{diag}\left\{L_{u f}\right\}, L_{g}=\operatorname{diag}\left\{L_{u g}\right\} .
$$

Assume that $x_{e}$ is equilibrium point of system $\Sigma_{1}$, let $y(t)=x(t)-x_{e}, \Sigma_{1}$ can be rewritten as:

$$
\Sigma_{3}:\left\{\begin{array}{l}
\dot{y}(t)=-D_{r(t)} y(t)+A_{r(t)} F(y(t))+B_{r(t)} G(y(t-\tau)) \\
y(\theta)=\phi_{y}(\theta), \theta \in[-\tau, 0]
\end{array}\right.
$$

where $F(y())=.\left[h_{1}\left(y_{1}(.)\right), h_{2}\left(y_{2}(.)\right), \ldots, h_{n}\left(y_{n}(.)\right)\right]^{T}$ and $G(y())=.\left[\kappa_{1}\left(y_{1}().\right)\right.$, $\left.\kappa_{2}\left(y_{2}().\right), \ldots, \kappa_{n}\left(y_{n}().\right)\right]^{T}$ with $h_{u}\left(y_{u}().\right)=f_{u}\left(y_{u}()+.x_{e}\right)-f_{u}\left(x_{e}\right)$ and $\kappa_{u}\left(y_{u}().\right)=$ $g_{u}\left(y_{u}()+.x_{e}\right)-g_{u}\left(x_{e}\right)$ for $u \in[1,2, \cdots, n], \phi_{y}(\theta)=\phi(\theta)-x_{e}$. Obviously, $h_{u}($.$) and$ $\kappa_{u}($.$) also satisfy the Assumption 1$.

Assume that $x_{1 e}$ is equilibrium point of system $\Sigma_{2}$, let $y(k)=x(k)-x_{1 e}, \Sigma_{2}$ can be rewritten as:

$$
\Sigma_{4}:\left\{\begin{array}{l}
y(k+1)=D_{r(k)} y(k)+A_{r(k)} F(y(k))+B_{r(k)} G(y(k-\beta)) \\
y(\theta)=\varphi_{y}(\theta), \theta \in[-\beta, 0]
\end{array}\right.
$$

where $\varphi_{y}(\theta)=\varphi(\theta)-x_{1 e}$.

Remark 1. For systems $\Sigma_{3}$ and $\Sigma_{4}$, according to assumption 1 and the analysis in [25], we know that the external input vector $J_{i}$ and $J_{p}$ are removed by $y(t)=x(t)-x_{e}$ and $y(k)=x(k)-x_{1 e}$.

Lemma 1. [7] If $D_{i} \succeq 0, A_{i} \succeq 0, B_{i} \succeq 0, J_{i} \succeq 0$ and Assumption 1 hold, the system $\Sigma_{1}$ and $\Sigma_{3}$ are positive for nonnegative initial state.

Lemma 2. If $D_{p} \succeq 0, A_{p} \succeq 0, B_{p} \succeq 0, J_{p} \succeq 0$ and Assumption 1 hold, the system $\Sigma_{2}$ and $\Sigma_{4}$ are positive for nonnegative initial state.

Definition 1. The system $\Sigma_{1}$ and $\Sigma_{3}$ are exponentially mean stable(EMS) if there exist two positive constants $\varepsilon$ and $\gamma$ such that

$$
E\left\{\|y(t)\|_{1}\right\}<\varepsilon E\left\{\|y(0)\|_{1}\right\} e^{-\gamma\left(t-t_{0}\right)}
$$

for any nonnegative initial condition.

Definition 2. The system $\Sigma_{2}$ and $\Sigma_{4}$ are EMS if there exist two positive constants $\varepsilon_{1}$ and $0<\xi<1$ such that

$$
E\left\{\|y(k)\|_{1}\right\}<\varepsilon_{1} E\left\{\|y(0)\|_{1}\right\} \xi^{\left(k-k_{0}\right)}
$$

for any nonnegative initial condition.

Lemma 3. [11] Consider a stochastic process $\{f(t), r(t), t \leq 0\}$ such that the jumping process $r(t)$ is a homogeneous Markov chain with right-continuous trajectories 
and takes in set $\mathbb{M}$. Assuming $E\left[f(t) \mathbf{1}_{r_{t}=i}\right]:=\boldsymbol{f}_{i}(t)$ exists, then $E\left[f(t) d\left(\mathbf{1}_{r_{t}=i}\right)\right]=$ $\sum_{j=1}^{M} \lambda_{j i} f_{j}(t) d t$, where

$$
\mathbf{1}_{\{r(t)=i\}} \begin{cases}1 & r(t)=i, \\ 0 & \text { otherwise. }\end{cases}
$$

When $\mathbb{M}=\{1\}$ and $\mathbb{N}=\{1\}$, the $\Sigma_{3}$ and $\Sigma_{4}$ are transformed into the continuous-time PNNs $\Gamma_{1}$ and the discrete-time PNNs $\Gamma_{2}$ respectively, as shown below

$$
\begin{gathered}
\Gamma_{1}:\left\{\begin{array}{l}
\dot{y}(t)=-D y(t)+A F(y(t))+B G(y(t-\tau)) \\
y(\theta)=\phi_{y}(\theta), \theta \in[-\tau, 0]
\end{array}\right. \\
\Gamma_{2}:\left\{\begin{array}{l}
y(k+1)=D y(k)+A F(y(k))+B G(y(k-\beta)) \\
y(\theta)=\varphi_{y}(\theta), \theta \in[-\beta, 0]
\end{array}\right.
\end{gathered}
$$

Definition 3. The system $\Gamma_{1}$ is exponentially stable(ES) if there exist two positive constants $\varepsilon$ and $\gamma$ such that

$$
\|y(t)\|_{1}<\varepsilon\|y(0)\|_{1} e^{-\gamma\left(t-t_{0}\right)}
$$

for any nonnegative initial condition.

Definition 4. The system $\Gamma_{2}$ are ES if there exist two positive constants $\varepsilon_{1}$ and $0<$ $\xi<1$ such that

$$
\|y(k)\|_{1}<\varepsilon_{1}\|y(0)\|_{1} \xi^{\left(k-k_{0}\right)}
$$

for any nonnegative initial condition.

\section{Main Results}

3.1 EMS of continuous-time PMJNNs

In this subsection, the continuous-time PMJNNs is rewritten as an equivalent continuoustime PNNs. By means of analyzing the exponential stability issue of the continuoustime PNNs, a sufficient condition is derived to ensure that the system $\Sigma_{3}$ is EMS.

For simplicity of presentation, according to Lemma 3, introduce the following notations:

$$
\begin{aligned}
& \mathbb{Y}=\left[\boldsymbol{y}_{1}^{T}(t) \boldsymbol{y}_{2}^{T}(t) \cdots \boldsymbol{y}_{M}^{T}(t)\right] \\
& \boldsymbol{y}_{i}(t)=E\left\{y(t) \mathbf{1}_{\{r(t)=i\}}\right\} \\
& F\left(\boldsymbol{y}_{i}(t)\right)=E\left\{F(y(t)) \mathbf{1}_{\{r(t)=i\}}\right\} \\
& \mathbb{D}=\operatorname{diag}\left\{D_{i}\right\}-\Upsilon^{T} \otimes I_{n}, \\
& \mathbb{A}=\operatorname{diag}\left\{A_{i}\right\}, \\
& \mathbb{B}=\operatorname{diag}\left\{B_{i}\right\}\left(\Omega^{T}(\tau) \otimes I_{n}\right)
\end{aligned}
$$


where $\Omega(\tau)=\left\{\rho_{i j}(\tau)\right\}$ and $\rho_{i j}(\tau)=\mathscr{P}_{r}\{r(t+\tau)=j \mid r(t)=i\}$ for $i, j \in \mathbb{M}, \tau \geq$ $0, t \geq 0$. And $\Omega(\tau)$ satisfies the forward Kolmogorov differential equation: $\dot{\Omega}(\tau)=$ $\Omega(\tau) r, \Omega(\tau)=I_{M}, \tau \geq 0$. It can obtain that $\Omega(\tau)=e^{r \tau}, \tau \geq 0$.

Theorem 1. Under Lemma 1 , if there exists a vector $\mathbb{P}=\left[P_{1}, P_{2}, \cdots, P_{M}\right]^{T} \in \mathbb{R}_{+}^{M n}$ and a constant $\gamma>0$ for $i, j \in \mathbb{M}$, such that

$$
\sum_{j=1}^{M} \lambda_{j i} P_{j}+\sum_{j=1}^{M} \rho_{j i}(\tau) e^{\gamma \tau} B_{i} L_{g} P_{j}+\left(A_{i} L_{f}+\gamma-D_{i}\right) P_{i} \prec 0
$$

then, the system $\Sigma_{3}$ is positive and EMS for a given $\tau$.

where $P_{i}=\left[o_{1}^{i}, o_{2}^{i}, \cdots, o_{c}^{i}\right]^{T}$ for $i \in \mathbb{M}$ and $c \in[1,2, \ldots, n]$.

Proof. From (4), for each $i, j \in \mathbb{M}$, we have

$$
\begin{aligned}
d \boldsymbol{y}_{i}(t)= & d E\left[y(t) \mathbf{1}_{r_{t}=i}\right]=E\left[d y(t) \mathbf{1}_{r_{t}=i}+y(t) d \mathbf{1}_{r_{t}=i}\right] \\
= & -D_{i} \boldsymbol{y}_{i}(t) d t+A_{i} F\left(\boldsymbol{y}_{i}(t)\right) d t+B_{i} E\left\{G(y(t-\tau)) \mathbf{1}_{r_{t}=i}\right\} d t+\sum_{j=1}^{M} \lambda_{j i} \boldsymbol{y}_{j}(t) d t \\
= & -D_{i} \boldsymbol{y}_{i}(t) d t+B_{i} \sum_{j=1}^{M} \mathscr{P}_{r}\left\{r_{t}=i \mid r_{t-\tau}=j\right\} E\left\{G(y(t-\tau)) \mathbf{1}_{r_{t-\tau}=j}\right\} d t \\
& +A_{i} F\left(\boldsymbol{y}_{i}(t)\right) d t+\sum_{j=1}^{M} \lambda_{j i} \boldsymbol{y}_{j}(t) d t \\
= & -D_{i} \boldsymbol{y}_{i}(t) d t+A_{i} F\left(\boldsymbol{y}_{i}(t)\right) d t+B_{i} \sum_{j=1}^{M} \rho_{j i}(\tau) G\left(\boldsymbol{y}_{j}(t-\tau)\right) d t \\
& +\sum_{j=1}^{M} \lambda_{j i} \boldsymbol{y}_{j}(t) d t
\end{aligned}
$$

that is

$$
\dot{\boldsymbol{y}}_{i}(t)=-D_{i} \boldsymbol{y}_{i}(t)+A_{i} F\left(\boldsymbol{y}_{i}(t)\right)+B_{i} \sum_{j=1}^{M} \rho_{j i}(\beta) G\left(\boldsymbol{y}_{j}(t-\beta)\right)+\sum_{j=1}^{M} \lambda_{j i} \boldsymbol{y}_{j}(t)
$$

On the basis of (5), (8) is written in vector form as follows

$$
\Sigma_{5}:\left\{\begin{array}{l}
\dot{\mathbb{Y}}(t)=-\mathbb{D} \mathbb{Y}(t)+\mathbb{A} \mathbb{F}(\mathbb{Y}(t))+\mathbb{B} \mathbb{G}(\mathbb{Y}(t-\tau)) \\
\mathbb{Y}(\theta)=\phi_{y}(\theta), \theta \in[-\tau, 0]
\end{array}\right.
$$

where $\mathbb{F}(\mathbb{Y}(t))=\left[F\left(\boldsymbol{y}_{1}(t)\right), F\left(\boldsymbol{y}_{2}(t)\right), \cdots,\left(\boldsymbol{y}_{M}(t)\right)\right], \mathbb{G}(\mathbb{Y}(t-\tau))=\left[G\left(\boldsymbol{y}_{1}(t-\tau)\right)\right.$, $\left.G\left(\boldsymbol{y}_{2}(t-\tau)\right), \cdots, G\left(\boldsymbol{y}_{M}(t-\tau)\right)\right]$. Clearly, the activation functions $\mathbb{F}($.$) and \mathbb{G}($.$) are$ continuous on $R^{M n}$ and satisfy Assumption 1 . We define

$$
\mathbb{L}_{f}=\operatorname{diag}\left\{L_{f}\right\}, \mathbb{L}_{g}=\operatorname{diag}\left\{L_{g}\right\}
$$

Then

$$
\|\mathbb{Y}(t)\|_{1}=\mathbf{1}_{M n}^{T}(\mathbb{Y}(t))=\mathbf{1}_{n}^{T}\left(\sum_{i=1}^{M}\left(\boldsymbol{y}_{i}(t)\right)\right)=E\left\{\|y(t)\|_{1}\right\}
$$


From (10) and Definition 3, we know that there exist positive constants $\varepsilon$ and $\gamma$ such that

$$
E\left[\|y(t)\|_{1}\right]=\|\mathbb{Y}(t)\|_{1}<\varepsilon\|\mathbb{Y}(0)\|_{1} e^{-\gamma\left(t-t_{0}\right)}=\varepsilon E\left[\|y(0)\| \|_{1}\right] e^{-\gamma\left(t-t_{0}\right)}
$$

So, from Definition 1, we know that the system $\Sigma_{3}$ is EMS if the system $\Sigma_{5}$ is ES.

Next, we will discuss the exponential stability of the system $\Sigma_{5}$. Under Lemma 1 , let $\mathbb{Z}(t)=e^{\gamma\left(t-t_{0}\right)} \mathbb{Y}(t)$, taking the upper right derivative of $\mathbb{Z}(t)$, we obtain

$$
\begin{aligned}
D^{+} \mathbb{Z}(t) & =e^{\gamma\left(t-t_{0}\right)}\{-\mathbb{D} \mathbb{Y}(t)+\mathbb{A} \mathbb{F}(\mathbb{Y}(t))+\mathbb{B} \mathbb{G}(\mathbb{Y}(t-\tau))\}+\gamma e^{\gamma\left(t-t_{0}\right)} \mathbb{Y}(t) \\
& \preceq e^{\gamma\left(t-t_{0}\right)}\left\{-\mathbb{D} \mathbb{Y}(t)+\mathbb{A} \mathbb{L}_{f} \mathbb{Y}(t)+\mathbb{B} \mathbb{L}_{g} \mathbb{Y}(t-\tau)\right\}+\gamma e^{\gamma\left(t-t_{0}\right)} \mathbb{Y}(t) \\
& =e^{\gamma\left(t-t_{0}\right)}\left\{(-\mathbb{D}+\boldsymbol{\sigma}) \mathbb{Y}(t)+\mathbb{A L}_{f} \mathbb{Y}(t)+\mathbb{B L}_{g} \mathbb{Y}(t-\tau)\right\}
\end{aligned}
$$

where $\boldsymbol{\sigma}=\gamma I_{M n}$. After substituting $\mathbb{Z}(t)$ for $e^{\gamma\left(t-t_{0}\right)} \mathbb{Y}(t)$ in Equ.(12), the following formula is obtained

$$
D^{+} \mathbb{Z}(t)=(-\mathbb{D}+\boldsymbol{\sigma}) \mathbb{Z}(t)+\mathbb{A} \mathbb{L}_{f} \mathbb{Z}(t)+e^{\gamma \tau} \mathbb{B L}_{g} \mathbb{Z}(t-\tau)
$$

Defining a curve in $M n$-dimensional space $\chi=\left\{\mathbb{H}(l): H_{i}=o_{i}^{c} l, l>0, i=1,2, \cdots\right.$, $M, c=1,2, \cdots, n\}$ and a set $\Lambda(\mathbb{H})=\{\mathbb{Z}: 0 \preceq \mathbb{Z} \preceq \mathbb{H}, \mathbb{H} \in \chi\}$. Let $l_{0}=\|\mathbb{Y}(0)\|_{1} / o_{\text {min }}$, where $o_{\text {min }}=\min _{1 \leq i \leq M ; 1 \leq c \leq n}\left\{o_{c}^{i}\right\}>0$. It is clear that $\Lambda\left(\mathbb{H}\left(l^{\prime}\right)\right) \subset \Lambda(\mathbb{H}(l))$ as $l>l^{\prime}$. Defining the $\mathbb{Z}(0)=e^{-\gamma t_{0}} \mathbb{Y}(0)$, we know $\mathbb{Z}(0) \subset \Lambda\left(\mathbb{H}\left(l_{0}\right)\right)$. That is $\mathbb{Z}(0)=e^{-\gamma t_{0}} \mathbb{Y}(0) \prec$ $\mathbb{P} l_{0}$.

For $t>0$, we assume that $\mathbb{Z}(t) \prec \mathbb{P} l_{0}$. If this is not true, then there are corresponding $t^{\prime}>0$, which make $\mathbb{Z}\left(t^{\prime}\right)=\mathbb{P} l_{0}, D^{+} \mathbb{Z}\left(t^{\prime}\right) \succeq 0$. According to (13), we get

$$
\begin{aligned}
D^{+} \mathbb{Z}\left(t^{\prime}\right) & \preceq(-\mathbb{D}+\boldsymbol{\sigma}) \mathbb{P} l_{0}+\mathbb{A} \mathbb{L}_{f} \mathbb{P} l_{0}+e^{\gamma \tau} \mathbb{B} \mathbb{L}_{g} \mathbb{P} l_{0} \\
& \preceq\left(-\mathbb{D}+\boldsymbol{\sigma}+\mathbb{A} \mathbb{L}_{f}+e^{\gamma \tau} \mathbb{B} \mathbb{L}_{g}\right) \mathbb{P} l_{0}
\end{aligned}
$$

let

$$
W=\left(-\mathbb{D}+\boldsymbol{\sigma}+\mathbb{A L}_{f}+e^{\gamma \tau} \mathbb{B L}_{g}\right) \mathbb{P}
$$

Substituting (5) and (9) into (15) yields

$$
W=\left(\begin{array}{cclc}
A_{11} & \lambda_{21} I_{n}+B_{1} \rho_{21}(\tau) L_{g} & \cdots & \lambda_{M 1} I_{n}+B_{1} \rho_{M 1}(\tau) L_{g} \\
\lambda_{12} I_{n}+B_{2} \rho_{12}(\tau) L_{g} & A_{22} & \cdots & \lambda_{M 2} I_{n}+B_{2} \rho_{M 2}(\tau) L_{g} \\
\vdots & \vdots & \ddots & \vdots \\
\lambda_{1 M} I_{n}+B_{3} \rho_{1 M}(\tau) L_{g} & \lambda_{2 M} I_{n}+B_{M} \rho_{2 M}(\tau) L_{g} & \cdots & A_{M M}
\end{array}\right)\left(\begin{array}{c}
P_{1} \\
P_{2} \\
\vdots \\
P_{M}
\end{array}\right)
$$

where $A_{i i}=A_{i} L_{f}+\gamma I_{n}+\lambda_{i i} I_{n}-D_{i}+e^{\gamma \tau} B_{i} \rho_{i i}(\tau) L_{g}, i \in \mathbb{M}$. Then,

$$
W=\left(\begin{array}{c}
\sum_{j=1}^{M} \lambda_{j 1} P_{j}+\sum_{j=1}^{M} \rho_{j 1}(\tau) e^{\gamma \tau} B_{1} L_{g} P_{j}+\left(A_{1} L_{f}+\gamma-D_{1}\right) P_{1} \\
\sum_{j=1}^{M} \lambda_{j 2} P_{j}+\sum_{j=1}^{M} \rho_{j 2}(\tau) e^{\gamma \tau} B_{2} L_{g} P_{j}+\left(A_{2} L_{f}+\gamma-D_{i}\right) P_{2} \\
\vdots \\
\sum_{j=1}^{M} \lambda_{j 3} P_{j}+\sum_{j=1}^{M} \rho_{j 3}(\tau) e^{\gamma \tau} B_{3} L_{g} P_{j}+\left(A_{3} L_{f}+\gamma-D_{i}\right) P_{3}
\end{array}\right)
$$


From (6) and (17), it is easy to get $W=\left(-\mathbb{D}+\boldsymbol{\sigma}+\mathbb{A L}_{f}+e^{\gamma \tau} \mathbb{B L}_{g}\right) \mathbb{P} \prec 0$, which means $D^{+} \mathbb{Z}\left(t^{\prime}\right) \prec 0$. Obviously, this is a contradiction. Thus $\mathbb{Z}(t) \prec \mathbb{P} l_{0}$ for $t \geq 0$. That is

$$
\|\mathbb{Y}(t)\|_{1} \prec e^{-\gamma\left(t-t_{0}\right)}\|\mathbb{P}\|_{1} l_{0}=\frac{\|\mathbb{P}\|_{1}}{o_{\min }} E\left\{\|y(0)\|_{1}\right\} e^{-\gamma\left(t-t_{0}\right)}
$$

Then, we have

$$
E\left\{\|y(t)\|_{1}\right\}<\varepsilon E\left\{\|y(0)\|_{1}\right\} e^{-\gamma\left(t-t_{0}\right)}
$$

where $\varepsilon=M n \cdot \max _{1 \leq i \leq M, 1 \leq c \leq n},\left\{o_{c}^{i}\right\} / o_{\min }$. The proof is completed.

\subsection{EMS of discrete-time PMJNNs}

In this subsection, the discrete-time PMJNNs is overwritten as an equivalent discretetime PNNs. Through the discussion on the exponential stability issue of the discretetime PNNs, a sufficient condition for the EMS of the system $\Sigma_{4}$ is provided.

First, define the indicator function [10]

$$
\mathbf{1}_{\{r(k)=i\}}(\omega) \begin{cases}1 & \text { if } r(k)(\omega)=i, i \in \mathbb{M} \\ 0 & \text { otherwise }\end{cases}
$$

and introduce the following notations

$$
\begin{aligned}
& \mathbb{Y}(k)=\left[\boldsymbol{y}_{1}^{T}(k) \boldsymbol{y}_{2}^{T}(k) \cdots \boldsymbol{y}_{N}^{T}(k)\right], \\
& \boldsymbol{y}_{p}(k)=E\left\{y(k) \mathbf{1}_{\{r(k)=p\}}\right\} \\
& F\left(\boldsymbol{y}_{p}(k)\right)=E\left\{F(y(k)) \mathbf{1}_{\{r(k)=p\}}\right\} \\
& \mathbb{D}=\left(\Pi^{T} \otimes I_{n}\right) \operatorname{diag}\left\{D_{p}\right\}, \\
& \mathbb{A}=\left(\Pi^{T} \otimes I_{n}\right) \operatorname{diag}\left\{A_{p}\right\} \\
& \mathbb{B}=\left(\Pi^{T} \otimes I_{n}\right) \operatorname{diag}\left\{B_{p}\right\}\left(\Psi^{T}(\beta) \otimes I_{n}\right)
\end{aligned}
$$

where $\Psi(\beta)=\left\{\delta_{p q}(\beta)\right\}$ and $\delta_{p q}(\beta)=\mathscr{P}_{r}\{\{r(k+\beta)=q \mid r(k)=p\}$ for $p, q \in \mathbb{N}, \beta \geq$ $0, k \geq 0$. And $\Psi(\beta)$ satisfies the equation $\Psi(\beta)=\Pi^{\beta}$.

Theorem 2. Under Lemma 2 , if there exists a vector $\mathbb{P}=\left[P_{1}, P_{2}, \ldots, P_{N}\right]^{T} \in \mathbb{R}_{+}^{N n}$ and a positive constant $0<\xi<1$ for $p, q, \eta \in \mathbb{N}$, such that

$$
\xi^{-1}\left\{\sum_{p=1}^{N} \pi_{p q} D_{p} P_{p}+\sum_{p=1}^{N} \pi_{p q} A_{p} L_{f} P_{p}+\xi^{-\beta} \sum_{p=1}^{N} \pi_{p q} B_{p} \sum_{\eta=1}^{N} \delta_{\eta p} L_{g} P_{\eta}\right\}-P_{q} \prec 0
$$

then, the system $\Sigma_{4}$ is positive and EMS for a given $\beta$. where $P_{p}=\left[o_{1}^{p}, o_{2}^{p}, \cdots, o_{c}^{p}\right]^{T}$ for $p \in \mathbb{N}$ and $c \in[1,2, \ldots, n]$. 
Proof. For each $p, q \in \mathbb{N}$, we have

$$
\begin{aligned}
\boldsymbol{y}_{q}(k+1)= & E\left\{y(k+1) \mathbf{1}_{r_{k+1}=q}\right\} \\
= & \sum_{p=1}^{N} \pi_{p q} D_{p} E\left\{y(k) \mathbf{1}_{r_{k}=p}\right\}+\sum_{p=1}^{M} \pi_{p q} A_{p} E\left\{F\left(y(k) \mathbf{1}_{r_{k}=p}\right\}\right. \\
& +\sum_{p=1}^{N} \pi_{p q} B_{p} E\left\{G\left(y(k-\beta) \mathbf{1}_{r_{k}=p}\right\}\right. \\
= & \sum_{p=1}^{N} \pi_{p q} D_{p} \boldsymbol{y}_{p}(k)+\sum_{p=1}^{N} \pi_{p q} A_{p} F\left(\boldsymbol{y}_{p}(k)\right) \\
& +\sum_{p=1}^{N} \pi_{p q} B_{p} \sum_{\eta=1}^{N} \mathscr{P}_{r}\{r(k)=p \mid r(k-\beta)=\eta\} E\left\{G(y(k-\beta)) \mathbf{1}_{r_{(k-\beta)}=\eta}\right\} \\
= & \sum_{p=1}^{N} \pi_{p q} D_{p} \boldsymbol{y}_{p}(k)+\sum_{p=1}^{N} \pi_{p q} A_{p} F\left(\boldsymbol{y}_{p}(k)\right) \\
& +\sum_{p=1}^{N} \pi_{p q} B_{p} \sum_{\eta=1}^{N} \delta_{\eta p} G\left(\boldsymbol{y}_{\eta}(k-\beta)\right)
\end{aligned}
$$

Write it in vector form as follows

$$
\Sigma_{6}:\left\{\begin{array}{l}
\mathbb{Y}(k+1)=\mathbb{D} \mathbb{Y}(k)+\mathbb{A} \mathbb{F}(\mathbb{Y}(k))+\mathbb{B} \mathbb{G}(\mathbb{Y}(k-\beta)) \\
\mathbb{Y}(\theta)=\boldsymbol{\varphi}_{y}(\theta), \theta \in[-\beta, 0]
\end{array}\right.
$$

where $\mathbb{F}(\mathbb{Y}(k))=\left[F\left(\boldsymbol{y}_{1}(k)\right), F\left(\boldsymbol{y}_{2}(k)\right), \cdots,\left(\boldsymbol{y}_{N}(k)\right)\right], \mathbb{G}(\mathbb{Y}(k-\beta))=\left[G\left(\boldsymbol{y}_{1}(k-\beta)\right)\right.$, $\left.G\left(\boldsymbol{y}_{2}(k-\beta)\right), \cdots, G\left(\boldsymbol{y}_{N}(k-\beta)\right)\right]$. According to (10), Definition 2 and Definition 4, we know that the systems $\Sigma_{4}$ is EMS if the system $\Sigma_{6}$ is ES.

Next, we will discuss exponential stability issue of the system $\Sigma_{6}$. Under Lemma 2 , let $\mathbb{Z}(k)=\xi^{-\left(k-k_{0}\right)} \mathbb{Y}(k)$, we have

$$
\begin{aligned}
\mathbb{Z}(k+1) & =\xi^{-\left(k+1-k_{0}\right)} \mathbb{Y}(k+1) \\
& \preceq \xi^{-\left(k+1-k_{0}\right)}\{\mathbb{D} \mathbb{Y}(k)+\mathbb{A} \mathbb{F}(\mathbb{Y}(k))+\mathbb{B} \mathbb{G}(\mathbb{Y}(k-\beta))\} \\
& \preceq \xi^{-1} \xi^{-\left(k-k_{0}\right)}\left\{\mathbb{D} \mathbb{Y}(k)+\mathbb{A L}_{f} \mathbb{Y}(k)+\mathbb{B} \mathbb{L}_{g} \mathbb{Y}(k-\beta)\right\}
\end{aligned}
$$

Substituting $\mathbb{Z}(k)=\xi^{-\left(k-k_{0}\right)} \mathbb{Y}(k)$ into $(25)$ gives

$$
\mathbb{Z}(k+1) \preceq \xi^{-1}\left\{\mathbb{D} \mathbb{Z}(k)+\mathbb{A} \mathbb{L}_{f} \mathbb{Z}(k)+\xi^{-\beta} \mathbb{B L}_{g} \mathbb{Z}(k-\beta)\right\}
$$

Defining a curve in $N n$-dimensional space $\vartheta=\left\{\mathbb{E}(l): E_{p}=o_{p}^{c} l, l>0, p=1,2, \cdots, N, c=\right.$ $1,2, \cdots, n\}$ and a set $\Lambda(\mathbb{E})=\{\mathbb{Z}: 0 \preceq \mathbb{Z} \preceq \mathbb{E}, \mathbb{E} \in \vartheta\}$. Let $l_{0}=\|\mathbb{Y}(0)\| / o_{\text {min }}$, where $o_{\text {min }}=\min _{1 \leq p \leq N, 1 \leq c \leq n}\left\{o_{c}^{p}\right\}$. It is clear that $\Lambda\left(\mathbb{E}\left(l^{\prime}\right)\right) \subset \Lambda(\mathbb{E}(l))$ as $l>l^{\prime}$. Defining the $\mathbb{Z}(0)=\xi^{k_{0}} \mathbb{Y}(0)$, we know $\mathbb{Z}(0) \subset \Lambda\left(\mathbb{E}\left(l_{0}\right)\right)$. That is $\mathbb{Z}(0)=\xi^{k_{0}} \mathbb{Y}(0) \prec \mathbb{P} l_{0}$. 
For $k>0$, we assert that $\mathbb{Z}(k)=\xi^{-\left(k-k_{0}\right)} \mathbb{Y}(k) \prec \mathbb{P} l_{0}$. If this assertion is invalid, then there must be a corresponding $k^{\prime}>0$ which makes $\mathbb{Z}\left(k^{\prime}\right)=\mathbb{P} l_{0}$ and $\Delta \mathbb{Z}\left(k^{\prime}\right) \succeq 0$ hold. Nevertheless, from (26), we know that

$$
\begin{aligned}
\Delta \mathbb{Z}\left(k^{\prime}\right) & =\mathbb{Z}\left(k^{\prime}+1\right)-\mathbb{Z}\left(k^{\prime}\right) \\
& \preceq \xi^{-1}\left\{\mathbb{D} \mathbb{Z}\left(k^{\prime}\right)+\mathbb{A} \mathbb{L}_{f} \mathbb{Z}\left(k^{\prime}\right)+\xi^{-\beta} \mathbb{B} \mathbb{L}_{g} \mathbb{Z}\left(k^{\prime}-\beta\right)\right\}-\mathbb{Z}\left(k^{\prime}\right) \\
& \preceq\left\{\xi^{-1}\left\{\mathbb{D}+\mathbb{A L}_{f}+\xi^{-\beta} \mathbb{B} \mathbb{L}_{g}\right\} \mathbb{P}-\mathbb{P}\right\} l_{0}
\end{aligned}
$$

Let

$$
W_{1}=\xi^{-1}\left\{\mathbb{D}+\mathbb{A L}_{f}+\xi^{-\beta} \mathbb{B L}_{g}\right\} \mathbb{P}-\mathbb{P}
$$

Substituting (9) and (22) into (28) yields

$$
W_{1}=\xi^{-1}\left(\begin{array}{cccc}
Q_{11} & Q_{12} & \cdots & Q_{1 N} \\
Q_{21} & Q_{22} & \cdots & Q_{2 N} \\
\vdots & \vdots & \ddots & \vdots \\
Q_{N 1} & Q_{N 2} & \cdots & Q_{N N}
\end{array}\right)\left(\begin{array}{c}
P_{1} \\
P_{2} \\
\vdots \\
P_{N}
\end{array}\right)-\left(\begin{array}{c}
P_{1} \\
P_{2} \\
\vdots \\
P_{N}
\end{array}\right)
$$

where

$$
\begin{aligned}
& Q_{11}=\pi_{11} D_{1}+\pi_{11} A_{1} L_{f}+\xi^{-\beta}\left(\pi_{11} B_{1} \delta_{11} L_{g}+\pi_{21} B_{2} \delta_{12} L_{g}+\pi_{N 1} B_{N} \delta_{1 N} L_{g}\right) \\
& Q_{12}=\pi_{21} D_{2}+\pi_{21} A_{2} L_{f}+\xi^{-\beta}\left(\pi_{11} B_{1} \delta_{21} L_{g}+\pi_{21} B_{2} \delta_{22} L_{g}+\pi_{N 1} B_{N} \delta_{2 N} L_{g}\right) \\
& Q_{1 N}=\pi_{N 1} D_{N}+\pi_{N 1} A_{N} L_{f}+\xi^{-\beta}\left(\pi_{11} B_{1} \delta_{N 1} L_{g}+\pi_{21} B_{2} \delta_{N 2} L_{g}+\pi_{N 1} B_{N} \delta_{N N} L_{g}\right) \\
& Q_{21}=\pi_{12} D_{1}+\pi_{12} A_{1} L_{f}+\xi^{-\beta}\left(\pi_{12} B_{1} \delta_{11} L_{g}+\pi_{22} B_{2} \delta_{12} L_{g}+\pi_{N 2} B_{N} \delta_{1 N} L_{g}\right) \\
& Q_{22}=\pi_{22} D_{2}+\pi_{22} A_{2} L_{f}+\xi^{-\beta}\left(\pi_{12} B_{1} \delta_{21} L_{g}+\pi_{22} B_{2} \delta_{22} L_{g}+\pi_{N 2} B_{N} \delta_{2 N} L_{g}\right) \\
& Q_{2 N}=\pi_{N 2} D_{N}+\pi_{N 2} A_{N} L_{f}+\xi^{-\beta}\left(\pi_{12} B_{1} \delta_{N 1} L_{g}+\pi_{22} B_{2} \delta_{N 2} L_{g}+\pi_{N 2} B_{N} \delta_{N N} L_{g}\right) \\
& Q_{N 1}=\pi_{1 N} D_{1}+\pi_{1 N} A_{1} L_{f}+\xi^{-\beta}\left(\pi_{1 N} B_{1} \delta_{11} L_{g}+\pi_{2 N} B_{2} \delta_{12} L_{g}+\pi_{N N} B_{N} \delta_{1 N} L_{g}\right) \\
& Q_{N 2}=\pi_{2 N} D_{2}+\pi_{2 N} A_{2} L_{f}+\xi^{-\beta}\left(\pi_{1 N} B_{1} \delta_{21} L_{g}+\pi_{2 N} B_{2} \delta_{22} L_{g}+\pi_{N N} B_{N} \delta_{2 N} L_{g}\right) \\
& Q_{N N}=\pi_{N N} D_{N}+\pi_{N N} A_{N} L_{f}+\xi^{-\beta}\left(\pi_{1 N} B_{1} \delta_{N 1} L_{g}+\pi_{2 N} B_{2} \delta_{N 2} L_{g}+\pi_{N N} B_{N} \delta_{N N} L_{g}\right) \\
& T
\end{aligned}
$$

Then

$$
W_{1}=\left(\begin{array}{c}
\xi^{-1}\left\{\Xi_{1}+\sum_{p=1}^{N} \pi_{p 1} A_{p} L_{f} P_{p}+\xi^{-\beta} \sum_{p=1}^{N} \pi_{p 1} B_{p} \sum_{\eta=1}^{N} \delta_{\eta p} L_{g} P_{\eta}\right\}-P_{1} \\
\xi^{-1}\left\{\Xi_{2}+\sum_{p=1}^{N} \pi_{p 2} A_{p} L_{f} P_{p}+\xi^{-\beta} \sum_{p=1}^{N} \pi_{p 2} B_{p} \sum_{\eta=1}^{N} \delta_{\eta p} L_{g} P_{\eta}\right\}-P_{2} \\
\vdots \\
\xi^{-1}\left\{\Xi_{N}+\sum_{p=1}^{N} \pi_{p N} A_{p} L_{f} P_{p}+\xi^{-\beta} \sum_{p=1}^{N} \pi_{p N} B_{p} \sum_{\eta=1}^{N} \delta_{\eta p} L_{g} P_{\eta}\right\}-P_{N}
\end{array}\right)
$$

where $\Xi_{q}=\sum_{p=1}^{N} \pi_{p q} D_{p} P_{p}, q \in \mathbb{N}$.

From (23) and (30), it's obvious that $W_{1}=\xi^{-1}\left\{\mathbb{D}+\mathbb{A L}_{f}+\xi^{-\beta} \mathbb{B L}_{g}\right\} \mathbb{P}-\mathbb{P} \prec 0$ is established, which means $\Delta \mathbb{Z}\left(k^{\prime}\right) \prec 0$. It's easy to see that this is a contradiction to the previous assertion. So, $\mathbb{Z}(k)=\xi^{-\left(k-k_{0}\right)} \mathbb{Y}(k) \prec \mathbb{P} l_{0}$ for $k \geq 0$ holds.

Consequently

$$
\|\mathbb{Y}(k)\|_{1} \prec \xi^{\left(k-k_{0}\right)}\|\mathbb{P}\|_{1} l_{0}=\frac{\|\mathbb{P}\|_{1}}{o_{\min }}\|\mathbb{Y}(0)\|_{1} \xi^{\left(k-k_{0}\right)}
$$

Let $\varepsilon_{1}=N n \cdot \max _{1 \leq p \leq N, 1 \leq c \leq n}\left\{o_{c}^{p}\right\} / o_{\min }$, we get

$$
E\left\{\|y(k)\|_{1}\right\} \prec \varepsilon_{1} E\left\{\|y(0)\|_{1}\right\} \xi^{\left(k-k_{0}\right)}
$$


The proof is complete.

Remark 2. In this paper, by utilizing the special properties of the PMJNNs itself, the augmentation system approach is applied to solve the stability problem of PMJNNs with time-delay, which can reduce the complexity of system analysis. The EMS conditions in terms of the standard linear programming are derived in Theorem 1 and Theorem 2. Although stability conditions for PMJSSs have been discussed in [25], there are only sufficient conditions in the form of LMI. Thus, the conservatism of the results in [25] is greatly reduced by the proposed method in the paper.

Remark 3. Delay-dependent EMS conditions for continuous time PMJNNs and discrete time PMJNNs are given in Theorem 1 and Theorem 2. In this paper, the stability of the PMJNNS are directly affected by time delays, parameters $\gamma$ and $\xi$, which also can be verified by the example at numerical example section.

\section{Numerical example}

Example 1. Consider a class of the system $\Sigma_{3}$ with three operation modes described as follows:

$$
\begin{aligned}
& A_{1}=\left[\begin{array}{lll}
0.30 & 0.12 & 0.45 \\
0.56 & 0.25 & 0.30 \\
0.60 & 0.20 & 0.80
\end{array}\right], B_{1}=\left[\begin{array}{lll}
0.05 & 0.10 & 0.03 \\
0.10 & 0.08 & 0.04 \\
0.15 & 0.06 & 0.10
\end{array}\right], \\
& A_{2}=\left[\begin{array}{lll}
0.55 & 0.60 & 0.30 \\
1.20 & 0.50 & 0.28 \\
1.00 & 0.55 & 0.35 \\
1.30 & 0.65 & 0.80 \\
0.40 & 0.75 & 1.00 \\
0.26 & 0.68 & 1.10
\end{array}\right], B_{2}=\left[\begin{array}{lll}
0.06 & 0.02 & 0.15 \\
0.05 & 0.04 & 0.17 \\
0.15 & 0.27 & 0.36 \\
0.12 & 0.25 & 0.09 \\
0.39 & 0.33 & 0.22 \\
0.12 & 0.30 & 0.18
\end{array}\right], \\
& A_{3}=\left[\begin{array}{ccc}
0.75 & 0 & 0 \\
0 & 1 & 0 \\
0 & 0.8 & 0 \\
0 & 0 & 1.6 \\
1.5 & 0 & 0 \\
0 & 0.7 & 0 \\
0 & 0 & 0.9
\end{array}\right], \\
& D_{1}=,
\end{aligned}
$$

And the transition probability matrix $\Upsilon$ is given as below

$$
\Upsilon=\left[\begin{array}{ccc}
-0.6 & 0.2 & 0.4 \\
0.8 & -2 & 1.2 \\
0.7 & 1.5 & -2.2
\end{array}\right]
$$

In this paper, boltzmann sigmoid activation functions are selected as: $h_{u}\left(y_{u}\right)=$ $\frac{1-e^{\frac{-y_{u}}{\theta_{u f}}}}{1+e^{\frac{-y_{u}}{\theta_{u f}}}}, \kappa_{u}\left(y_{u}\right)=\frac{1-e^{\frac{-y_{u}}{\theta_{u g}}}}{1+e^{\frac{-y u}{\theta_{u g}}}}$. Parameter $\gamma$ and time delay $\tau$ are given as 0.5 and 0.2 . Then, from Assumption 1, we can obtain: $L_{u f}=\frac{1}{2 \theta_{u f}}, L_{u g}=\frac{1}{2 \theta_{u g}}, \theta_{u f}>0, \theta_{u g}>0, i \in$ M. Choosing the corresponding $\theta_{u f}$ and $\theta_{u g}$, we get

$$
\begin{aligned}
& L_{f}=\operatorname{diag}\{0.5,0.12,0.2\}, \\
& L_{g}=\operatorname{diag}\{0.2,0.10,0.4\} .
\end{aligned}
$$


Solving the linear programming problem in Theorem 1, we get:

$$
\begin{aligned}
& P_{1}=[0.0016 ; 0.0043 ; 0.0018]^{T} ; \\
& P_{2}=[0.0011 ; 0.0020 ; 0.0014]^{T} ; \\
& P_{3}=[0.0010 ; 0.0021 ; 0.0012]^{T} .
\end{aligned}
$$

The initial states for the system $\Sigma_{3}$ is chosen as: $\phi_{y}(0)=[5 ; 2.5 ; 3.5]$. Fig. 1 shows mode evolution of the system $\Sigma_{3}$. Fig. 2 depicts the state trajectories of the system $\Sigma_{3}$, which can draw the conclusion that the systems $\Sigma_{3}$ is positive and EMS.

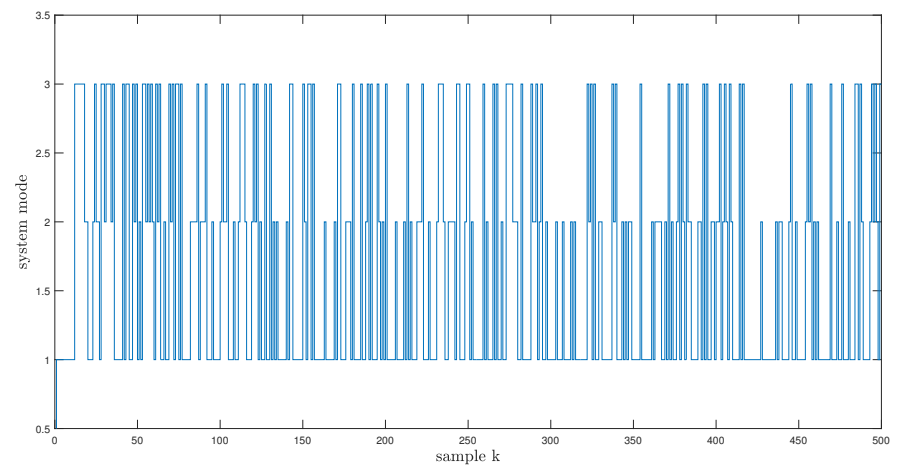

Fig. 1: System mode evolution in Example 1.

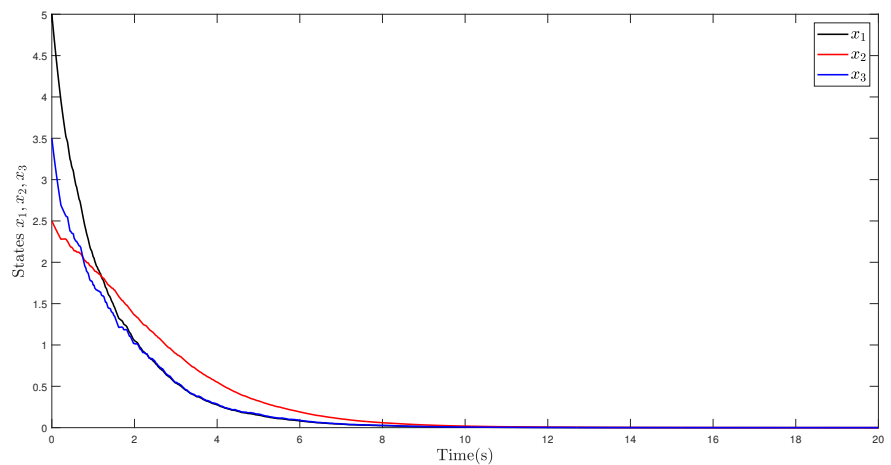

Fig. 2: System state evolution in Example 1.

Table 1 and Table 2 show the effects of different time delays $\tau$ and different system parameters $\gamma$ on the stability of the system $\Sigma_{3}$. 
Table 1: Comparison results for different time delay $\tau(\gamma=0.5)$

\begin{tabular}{|c|c|c|c|l|}
\hline$\tau$ & 0 & 0.1 & 0.2 & 0.3 \\
\hline Theorem 1 & Feasible & Feasible & Feasible & Feasible \\
\hline$\tau$ & 0.4 & 0.5 & 0.6 & 0.7 \\
\hline Theorem 1 & Feasible & Infeasible & Infeasible & Infeasible \\
\hline
\end{tabular}

Table 2: Comparison results for different system parameter $\gamma(\tau=0.2)$

\begin{tabular}{|c|c|c|c|l|}
\hline$\gamma$ & 0 & 0.1 & 0.2 & 0.3 \\
\hline Theorem 1 & Feasible & Feasible & Feasible & Feasible \\
\hline$\gamma$ & 0.4 & 0.5 & 0.6 & 0.7 \\
\hline Theorem 1 & Feasible & Feasible & Infeasible & Infeasible \\
\hline
\end{tabular}

Example 2. Consider a class of the system $\Sigma_{4}$ with three operation modes described as follows:

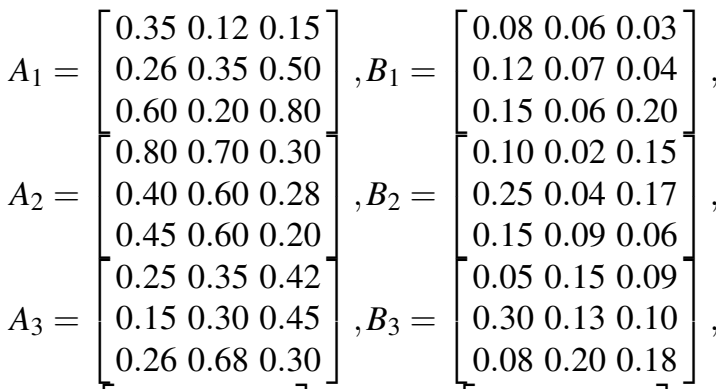

$$
\begin{aligned}
& D_{1}=\left[\begin{array}{ccc}
0.6 & 0 & 0 \\
0 & 0.4 & 0 \\
0 & 0 & 0.85
\end{array}\right], \quad D_{2}=\left[\begin{array}{ccc}
0.35 & 0 & 0 \\
0 & 0.6 & 0 \\
0 & 0 & 0.7
\end{array}\right] \text {, } \\
& D_{3}=\left[\begin{array}{ccc}
0.45 & 0 & 0 \\
0 & 0.8 & 0 \\
0 & 0 & 0.5
\end{array}\right] \text {. }
\end{aligned}
$$

And the transition probability matrix $\Pi$ is shown as

$$
\Pi=\left[\begin{array}{lll}
0.3 & 0.5 & 0.2 \\
0.4 & 0.3 & 0.3 \\
0.2 & 0.6 & 0.2
\end{array}\right]
$$

Similarly, by selecting the corresponding $\theta_{u f}$ and $\theta_{u g}$, we obtain

$$
\begin{aligned}
& L_{f}=\operatorname{diag}\{0.10,0.12,0.06\}, \\
& L_{g}=\operatorname{diag}\{0.20,0.10,0.15\} .
\end{aligned}
$$


The system parameter $\xi$ and time delay $\beta$ are selected as 0.82 and 2. Solving the linear programming problem in Theorem 2, we get:

$$
\begin{aligned}
& P_{1}=[0.0010 ; 0.0014 ; 0.0031]^{T} ; \\
& P_{2}=[0.0013 ; 0.0020 ; 0.0041]^{T} ; \\
& P_{3}=[0.0010 ; 0.0011 ; 0.0023]^{T} .
\end{aligned}
$$

The initial state is initialized to $\varphi_{y}(0)=[5 ; 2.5 ; 3.5]$. Fig. $3-4$ demonstrates the simulation results of the system $\Sigma_{4}$, which can illustrate that the systems $\Sigma_{4}$ is positive and EMS.

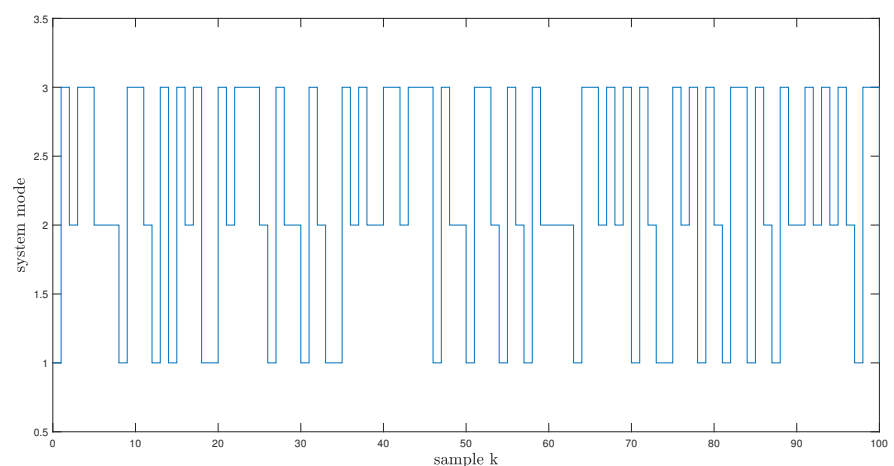

Fig. 3: System mode evolution in Example 2.

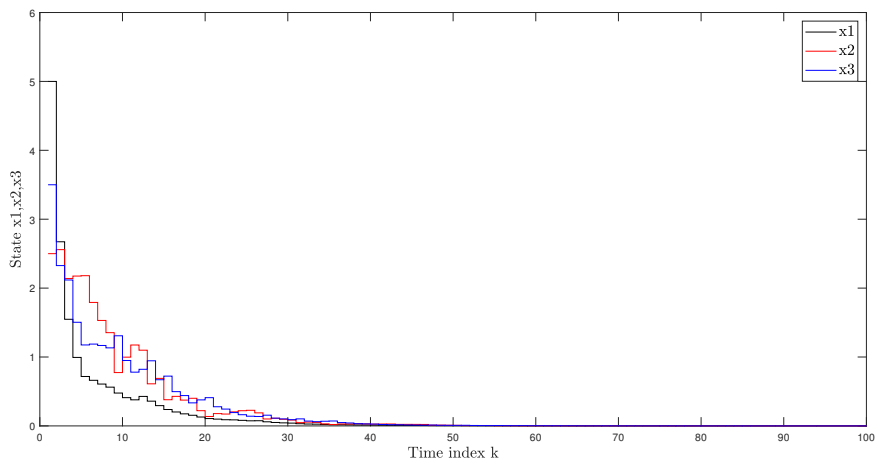

Fig. 4: System state evolution in Example 2.

Table 3 and Table 4 show the effects of different time delays $\beta$ and different system parameters $\xi$ on the stability of the system $\Sigma_{4}$. 
Table 3: Comparison results for different time delay $\beta(\xi=0.82)$

\begin{tabular}{|c|c|c|c|l|}
\hline$\beta$ & 0 & 1 & 2 & 3 \\
\hline Theorem 1 & Feasible & Feasible & Feasible & Infeasible \\
\hline$\beta$ & 4 & 5 & 6 & 7 \\
\hline Theorem 1 & Infeasible & Infeasible & Infeasible & Infeasible \\
\hline
\end{tabular}

Table 4: Comparison results for different system parameter $\xi(\beta=2)$

\begin{tabular}{|c|c|c|c|l|}
\hline$\gamma$ & 0.2 & 0.4 & 0.8 & 0.82 \\
\hline Theorem 1 & Infeasible & Infeasible & Infeasible & Feasible \\
\hline$\gamma$ & 0.86 & 0.90 & 0.94 & 0.98 \\
\hline Theorem 1 & Feasible & Feasible & Feasible & Feasible \\
\hline
\end{tabular}

\section{Conclusion}

In this paper, the linear programming method, the augmentation system method and inequality technique are used to investigate the EMS issues of PMJNNs with time delay. Sufficient criteria in the form of linear programming are put forward to guarantee the EMS on PMJNNs with time delay in both continuous-time domain and discretetime domain. Further more, this paper reveals that the EMS of PMJNNs with time delay is influenced by the size of time-delay. Two numerical examples is provided to prove the validity of our theoretical discovery. Finally, the effect of time-varying delay for positive Markov jump neural networks will be discussed in future work.

\section{Data Availability}

Data sharing is not applicable to this article as no new data were created or analyzed in this study.

\section{Declaration of Interest Statement}

The authors declare that they have no known competing financial interests or personal relationships that could have appeared to influence the work reported in this paper.

\section{References}

1. M. Galicki, H. Witte and J. Dorschel, Common optimization of adaptive preprocessing units and a neural network during the learning period Application in eeg pattern recognition, Neural Netw. 10 (6) (1997) 1153-1163.

2. Y. Pershin and M. Ventra, Experimental demonstration of associative memory with memristive neural networks, Neural Netw. 23 (7) (2010) 881-886.

3. B.K. Wong and Y. Selvi, Neural network applications in finance: a review of literature, Inf. Manag. 34 (3) (1998) 129-139.

4. L. Farina and S. Farina, "Positive linear systems, Theory and Applications", New York, NY, USA: WileyInterscience, 2000.

5. R. Shorten, F. Wirth, and D. Leith, A positive systems model of TCPlike congestion control: Asymptotic results, IEEE/ACM Trans. Netw. 14 (3) (2006) 616-629.

6. A. Rantzer, M. E. ValcherA, et.al., Tutorial on Positive Systems and Large Scale Control, IEEE Conference on Decision and Control (2018). 
7. L. V. Hien, On global exponential stability of positive neural networks with time-varying delay, Neural Netw. 87 (2017) 22-26.

8. L. V. Hien and L. D. Hai-An, Exponential stability of positive neural networks in bidirectional associative memory model with delays, Math. Methods Appl. Sci. 42 (18) (2019) 6339-6357.

9. S. Xiao, Y. Zhang and B. Zhang, $l_{1}$-gain filter design of discrete-time positive neural networks with mixed delays, Neural Netw. 122 (2020) 152-162.

10. O. Costa, M. Fragoso and R. Marques, Discrete-time Markov jump linear systems, London, U.K.: Springer-Verlag, (2005).

11. O. L. V. Costa, M, Fragoso and M. G. Todorov, Continuous-time Markov jump linear systems, Springer, London (2012)

12. D. Yao, B. Zhang and P. Li, Event-Triggered Sliding Mode Control of Discrete-Time Markov Jump Systems, IEEE Trans. Syst., Man, Cybern., Syst. 49 (10) (2019) 2016-2225.

13. M. Gao, L. Zhang and W. Qi, SMC for semi-Markov jump T-S fuzzy systems with time delay, Appl. Math. Comput. 374 (125001) (2020).

14. R. Li and J. Cao, Finite-Time Stability Analysis for Markovian Jump Memristive Neural Networks With Partly Unknown Transition Probabilities, IEEE Trans. Neural Netw. Learn. Syst. 28 (12) (2017) 2924-2935.

15. S. Jiao, H. Shen and Y. Wei, Further results on dissipativity and stability analysis of Markov jump generalized neural networks with time-varying interval delays, Appl. Math. Comput. 336 (2018) 338350 .

16. N. Xu and L. Sun, Synchronization control of Markov jump neural networks with mixed time-varying delay and parameter uncertain based on sample point controller, Nonlinear dynamics 98 (3) (2019) 1877-1890.

17. J. Wang and $\mathrm{H}$, Shen, Passivity-based fault-tolerant synchronization control of chaotic neural networks against actuator faults using the semi-Markov jump model approach, Neurocomputing 143 (2014) 51-56.

18. F. Li, S. Song and J. Zhao, Synchronization control for Markov jump neural networks subject to HMM observation and partially known detection probabilities, Appl. Math. Comput. 360 (2019) 1-13.

19. J. Tao, R. Lu and H. Su, Dissipativity-based asynchronous state estimation for Markov jump neural networks with jumping fading channels, Neurocomputing 241 (2017) 56-63.

20. X. Song, J. Man and Z. Fu, Memory-based State Estimation of T-S Fuzzy Markov Jump Delayed Neural Networks with Reaction-Diffusion Terms, Neural Process. Lett. 50 (3) (2019) 2529-2546.

21. H. Shen, S. Jiao and J. Xia, Generalised state estimation of Markov jump neural networks based on the Bessel-Legendre inequality, IET Control Theory Appl. 13 (9) (2019) 1284-1290.

22. M. Chen, $\mathrm{L}$. Zhang and $\mathrm{H}$. Shen, Resilient $\mathrm{H}$ infinity filtering for discrete-time uncertain Markov jump neural networks over a finite-time interval, Neurocomputing 185 (2016) 212-219.

23. Y. Shen, Z-G. Wu and P. Shi, Asynchronous Filtering for Markov Jump Neural Networks With Quantized Outputs, IEEE Trans. Syst., Man, Cybern., Syst. 49 (2) (2019) 433-443.

24. J. Tao, Z-G. Wu and H. Su, Asynchronous and Resilient Filtering for Markovian Jump Neural Networks Subject to Extended Dissipativity, IEEE Trans., Cybern. 49 (7) (2019) 2504-2513.

25. C. Ren and S, He, Finite-time stabilization for positive Markovian jumping neural networks, Appl. Math. Comput. 365 (2020)

26. S. Zhu, Q. Han, and C. Zhang, $l_{1}$-gain performance analysis and positive filter design for positive discrete-time Markov jump linear systems: A linear programming approach, Automatica 50 (8) (2014) 2098-2107. 